\section{Utilização de serviços de saúde pela população adulta de São Leopoldo, Rio Grande do Sul, Brasil: resultados de um estudo transversal}

\author{
Health services utilization by the adult population \\ in São Leopoldo, Rio Grande do Sul State, Brazil: \\ a cross-sectional study
}

Juvenal Soares Dias-da-Costa 1,2 Maria Teresa Anselmo Olinto 1 Simoni Assunção Soares 1 Marcelo Felipe Nunes 1 Tatiane Bagatini 1 Maximiliano das Chagas Marques 1 Lisiane Kiefer Guimarães 1 Letícia Possebon Müller 1 Fátima Carina de Souza Machado 1 Eduardo dos Santos Barcellos 1 Marcos Pascoal Pattussi 1

\section{Introdução}

The aim was to describe healthcare utilization by adults in a Brazilian city. The outcomes were medical appointments in the previous month and use of public (Unified National Health System SUS) versus private healthcare services. A population-based cross-sectional study with 1,098 adults aged 20 years or over was carried out. No medical appointment in the previous month was reported by 623 persons (56.7\%, 95\%CI: 53.8-59.7). Of the 487 individuals who had consulted a physician, $51.2 \%$ used the public healthcare system, 26.9\% private care, and $22 \%$ other services. Consultation was associated with female gender and older age. Individuals in the intermediate categories for income, schooling, and socioeconomic status consulted less than the corresponding high and low categories. The results suggest that the middle class in this city lacks the purchasing power to seek care in the private sector while also using public services less, thus generally seeking healthcare less frequently.

Health Services Accessibility; Health Evaluation; Equity in Health
As definições de sistemas de saúde que oferecem cuidados qualificados sempre incluem entre seus atributos o acesso facilitado aos serviços 1,2. Além disso, autores têm demonstrado que a oferta de cuidados primários em saúde é um aspecto importante para melhorar as condições de saúde da população ${ }^{3,4}$. Para Travassos \& Martins 5 (p. S192), “acesso é uma característica da oferta de serviços importante para a explicação do padrão de utilização de serviços de saúde", sendo que o uso compreende todo contato com os serviços de saúde.

Estudos epidemiológicos sobre serviços de saúde podem avaliar se o acesso universal, a integralidade dos cuidados ou se as bases da equidade estão sendo atingidos 6 .

Diversas pesquisas brasileiras, por exemplo, ainda revelam as iniquidades na utilização dos serviços de saúde, principalmente em relação às condições demográficas e socioeconômicas $7,8,9,10$. Estudos transversais de base populacional entre adultos realizados no Rio Grande do Sul têm mostrado que apesar da elevada utilização dos serviços de saúde, persistem marcadas diferenças entre indivíduos de acordo com características socioeconômicas. Na cidade de Rio Grande, por exemplo, foi mostrado que após ajuste para idade e sexo os grupos de menor renda e escolaridade tinham $62 \%$ menos probabilidade de usar serviços de saúde quando compa- 
rados com aqueles de maior renda 11. Estudos realizados em Pelotas encontraram diferenças socioeconômicas na utilização de serviços de saúde. Capilheira \& Santos 12 observaram na análise ajustada por sexo e idade que indivíduos inseridos nas classes econômicas A e B consultavam $13 \%$ mais. Outro estudo realizado na mesma cidade apontou que tanto os homens quanto as mulheres de baixa renda se consultavam menos nos serviços ambulatoriais 13. Em São Leopoldo, uma pesquisa incluindo apenas a população feminina apontou que as mulheres inseridas nas classes econômicas C, D e E, com baixa renda e pior nível de escolaridade, apresentavam maiores prevalências de não uso de serviços de saúde ${ }^{14}$. Assim, fica clara a importância da realização de estudos sobre o uso de serviços de saúde para a detecção e o apontamento de grupos populacionais excluídos das políticas de saúde.

O objetivo deste trabalho foi descrever o uso dos serviços de saúde e as características sociodemográficas associadas entre a população adulta do Município de São Leopoldo.

\section{Métodos}

Este é um estudo transversal realizado entre janeiro de 2006 e julho de 2007 com indivíduos de 20 a 69 anos, residentes na zona urbana do $\mathrm{Mu}$ nicípio de São Leopoldo.

São Leopoldo está situado na região do Vale do Rio dos Sinos, área metropolitana de Porto Alegre, e apresentava uma população estimada para 2007 de 207.721 habitantes (Instituto Brasileiro de Geografia e Estatística. http://www.ibge. gov.br/cidadesat/topwindow.htm/1, acessado em 02/Dez/2009). A Rede Municipal de Saúde está organizada em três níveis de atendimento: primário, com 28 unidades básicas fixas e móveis, oito Estratégia Saúde da Família cobrindo 18\% da população; nível secundário com cinco Centros de Saúde e um Serviço Ambulatorial Especializado; e terciário por meio do Hospital Centenário, além de um Centro de Atenção Psicossocial (CAPS Adulto) e dois CAPS em fase de implantação (Infantil e Álcool e outras Drogas).

Para se calcular o tamanho da amostra, considerou-se poder de $85 \%$ para se detectar uma diferença de 7\% na prevalência de autopercepção de saúde ruim entre áreas com alto e baixo capital social (principal desfecho estudado no projeto) e nível de 95\% de confiança. Estimando-se 35 domicílios por setor e coeficiente de correlação intraclasse igual a 0,05, o tamanho da amostra foi aumentado em $20 \%$ no número de domicílios e em $10 \%$ no número de setores devido a possíveis perdas e para controlar fatores de confusão. Portanto, foi necessário um total de 1.520 domicílios em 40 setores censitários dos 270 existentes em São Leopoldo. A amostragem foi por conglomerados.

O instrumento de coleta de dados foi um questionário padronizado e pré-codificado aplicado aos responsáveis pelos domicílios presentes no momento da entrevista. Os instrumentos foram aplicados por estudantes de graduação e pós-graduação da UNISINOS (Universidade do Vale do Rio dos Sinos), os quais receberam treinamento para realização das entrevistas. Os questionários e a logística foram testados em um estudo piloto realizado em outro setor não incluído no estudo. Por razões logísticas as entrevistas foram feitas durante o horário comercial.

Foram analisados três desfechos: não se consultar com médico no último mês, utilizar os serviços do SUS e se consultar nos serviços privados de saúde. Foi perguntado se os indivíduos haviam se consultado com médico nos últimos 30 dias. $\mathrm{O}$ desfecho inicialmente analisado foi não se consultar com médico no último mês. Entre os indivíduos que tinham se consultado foi indagado o local da consulta com três respostas possíveis: serviços do SUS, em serviços privados, e em outros serviços. Construíram-se duas variáveis dummies: utilizar os serviços do SUS e se consultar nos serviços privados de saúde.

As variáveis socioeconômicas analisadas foram: renda familiar (em salários mínimos), escolaridade (em anos de estudos), classe econômica. As variáveis renda familiar e escolaridade foram categorizadas em: alta $25 \%$ dos maiores escores dentre os indivíduos), média ( $50 \%$ dos escores intermediários) e baixa (25\% dos escores menores). A classificação de classe econômica da Associação Brasileira de Empresas de Pesquisas e Mercados (ABEP. Critérios de Classificação Econômica no Brasil. http://www.abep.org/codigi guias/criterio_Brasil_2008.pdf, acessado em 02/ Dez/2009) é um escore baseado no acúmulo de bens materiais e escolaridade.

As variáveis demográficas foram referentes a sexo, faixa etária, cor da pele observada pelo entrevistador (branca, preta/parda) e estado civil (casado, solteiro, outros).

A entrada de dados foi realizada no programa Epi Info 6 (Centers for Disease Control and Prevention, Atlanta, Estados Unidos) em dupla entrada e posterior comparação, para se eliminar a probabilidade de erros de digitação. A análise bruta foi realizada no programa SPSS, versão 17 (SPSS Inc., Chicago, Estados Unidos), descrevendo as razões de prevalência, os intervalos de 95\% de confiança (IC95\%) e testes estatísticos. Foi realizada análise ajustada, usando-se o programa Stata, versão 11 (Stata Corp., College Sta- 
tion, Estados Unidos), utilizando-se a regressão de Poisson com variância robusta ${ }^{15}$. Ingressaram na análise ajustada as variáveis que alcançaram valor de $\mathrm{p} \leq 0,20$ na análise bruta.

O projeto de pesquisa foi aprovado pelo Comitê de Ética em Pesquisa da UNISINOS em 2006 (CEP n ${ }^{\circ}$. 04/034).

\section{Resultados}

Foram realizadas 1.098 entrevistas em 38 setores, sendo que as perdas ocorreram por: recusas (4\%) e pessoas ausentes na residência $(2 \%)$.
Quanto à descrição das características dos indivíduos incluídos no estudo, observou-se que $56,2 \%$ foram classificados como de renda familiar média (mediana 5,1 salários mínimos), 65,2\% apresentavam escolaridade média (mediana 8,0 anos) e 45,9\% estavam inseridos na classe econômica C. Em relação às características demográficas, $71,9 \%$ eram mulheres, $23,5 \%$ estavam na faixa etária de 40-49 anos (mediana 44,0), 83,9\% foram classificados como de cor da pele branca e $55,9 \%$ eram casados (Tabela 1 ).

Entre as 1.098 pessoas respondentes, 623 (56,7\%; IC95\%: 53,8-59,7) não haviam se consultado com médico nos últimos 30 dias. Entre os

\section{Tabela 1}

Distribuição e prevalências de não se consultar com médicos no último mês, de acordo com as variáveis socioeconômicas e demográficas, em indivíduos de 20 a 69 anos, residentes na zona urbana do Município de São Leopoldo, Rio Grande do Sul, Brasil, 2006-2007 ( $N=1.100)$.

\begin{tabular}{|c|c|c|c|c|c|c|c|}
\hline \multirow[t]{2}{*}{ Variável } & \multirow[t]{2}{*}{ n } & \multirow[t]{2}{*}{$\%$} & \multicolumn{2}{|c|}{$\begin{array}{l}\text { Prevalência de } \\
\text { não consulta }\end{array}$} & \multirow[t]{2}{*}{$\begin{array}{c}\text { Razão de } \\
\text { prevalência }\end{array}$} & \multirow[t]{2}{*}{ IC95\% } & \multirow[t]{2}{*}{$\begin{array}{l}\text { Valor } \\
\text { de } p\end{array}$} \\
\hline & & & n & $\%$ & & & \\
\hline Renda familiar em salários mínimos [n = 1.074] & & & & & & & 0,57 \\
\hline Alta $(25 \%)$ & 268 & 25,0 & 140 & 52,2 & 1,00 & & \\
\hline Média (50\%) & 604 & 56,1 & 340 & 60,3 & 1,15 & $1,01-1,32$ & \\
\hline Baixa (25\%) & 203 & 18,9 & 111 & 54,3 & 1,04 & $0,88-1,22$ & \\
\hline Escolaridade (anos de estudos) [ $n=1.065]$ & & & & & & & 0,09 \\
\hline Alta (25\%) & 166 & 15,6 & 107 & 64,5 & 1,00 & & \\
\hline Média (50\%) & 695 & 65,2 & 383 & 55,1 & 0,85 & $0,75-0,97$ & \\
\hline Baixa (25\%) & 204 & 19,2 & 117 & 57,4 & 0,89 & $0,76-1,05$ & \\
\hline Classe econômica [n = 1.063] & & & & & & & 0,84 \\
\hline$A-B$ & 336 & 31,6 & 189 & 56,3 & 1,00 & & \\
\hline C & 488 & 45,9 & 283 & 58,0 & 1,03 & $0,91-1,16$ & \\
\hline$D-E$ & 239 & 22,4 & 134 & 56,1 & 1,00 & $0,86-1,15$ & \\
\hline Sexo $[n=1.098]$ & & & & & & & $<0,01$ \\
\hline Feminino & 790 & 71,9 & 429 & 54,3 & 1,00 & & \\
\hline Masculino & 308 & 28,1 & 194 & 63,0 & 1,16 & $1,04-1,29$ & \\
\hline Idade (anos) [n = 1.098] & & & & & & & 0,01 \\
\hline $20-29$ & 195 & 17,8 & 99 & 50,8 & 1,00 & & \\
\hline $30-39$ & 216 & 19,7 & 109 & 50,5 & 0,99 & $0,82-1,20$ & \\
\hline $40-49$ & 258 & 23,5 & 155 & 60,1 & 1,18 & $1,00-1,40$ & \\
\hline $50-59$ & 182 & 16,5 & 118 & 64,8 & 1,28 & $1,07-1,52$ & \\
\hline$\geq 60$ & 247 & 22,5 & 142 & 57,5 & 1,13 & $0,95-1,35$ & \\
\hline Cor da pele $[n=1.096]$ & & & & & & & 0,85 \\
\hline Branca & 920 & 83,9 & 521 & 56,6 & 1,00 & & \\
\hline Preta/Parda & 176 & 16,1 & 101 & 83,8 & 1,01 & $0,88-1,16$ & \\
\hline Estado civil [ $n=1.098$ ] & & & & & & & 0,1 \\
\hline Casado & 614 & 55,9 & 344 & 56,0 & 1,00 & & \\
\hline Solteiro & 267 & 24,3 & 165 & 61,8 & 1,10 & $0,98-1,24$ & \\
\hline Outros & 217 & 19,7 & 114 & 52,5 & 0,94 & $0,81-1,08$ & \\
\hline
\end{tabular}

IC95\%: intervalo de 95\% de confiança. 
485 indivíduos que tinham se consultado com médico no último mês, $51,2 \%$ utilizaram os serviços do SUS, $26,8 \%$ os serviços privados de saúde e $22 \%$ outros serviços.

$\mathrm{Na}$ análise bruta observaram-se os homens se consultando menos que as mulheres. A prevalência de não se consultar com médico foi $16 \%$ maior entre os homens. Os indivíduos de 40-59 anos também se consultaram menos. Os de renda familiar média se consultaram menos. Entretanto, os entrevistados com menor nível de escolaridade apresentaram menores prevalências de não consulta. Não foram observadas associações estatisticamente significativas em relação à renda, classe econômica da ABEP (valor de $\mathrm{p}=0,84$ ), cor da pele (valor de $\mathrm{p}=0,85$ ) e estado civil (valor de $\mathrm{p}=0,10$ ) (Tabela 1$)$.

Na análise ajustada de não consulta médica mantiveram associação com o desfecho as variáveis sexo, idade, escolaridade e renda familiar. Em relação à prevalência de não realização de consultas médicas no último mês, esta foi $21 \%$ maior entre os homens do que nas mulheres. Quanto à idade, os grupos entre 40-49 e 50-59 anos tiveram uma ocorrência do desfecho $19 \%$ e $25 \%$ maiores do que aqueles de 20-29 anos, respectivamente. Ainda, no grupo com escolaridade mais baixa, a prevalência de não consulta foi $12 \%$ menor do que naqueles com mais anos de estudos. Também, entre os indivíduos com renda familiar classificada como média, a ocorrência do desfecho foi $26 \%$ maior do que naqueles com renda mais alta (Tabela 2).

Os indivíduos de renda elevada se consultaram predominantemente nos serviços privados de saúde, enquanto aqueles inseridos na categoria de baixa renda majoritariamente procuraram o SUS (Figura 1).

Quanto ao uso de serviços do SUS, 77,9\% eram mulheres (valor de $\mathrm{p}=0,12$ ), $53 \%$ casados (valor de $\mathrm{p}=0,12$ ), 73,6\% possuíam escolaridade média (valor de $\mathrm{p}<0,001$ ), 54,6\% com renda

\section{Tabela 2}

Análise ajustada usando-se a Regressão de Poisson para não se consultar com médicos no último mês, de acordo com as variáveis socioeconômicas e demográficas, em indivíduos de 20 a 69 anos, residentes na zona urbana do Município de São Leopoldo, Rio Grande do Sul, Brasil, 2006-2007 ( $\mathrm{N}=1.100$ ).

\begin{tabular}{|c|c|c|c|}
\hline Variável & $\begin{array}{c}\text { Razão de prevalência } \\
\text { ajustada * }\end{array}$ & IC95\% & Valor de $p$ \\
\hline Renda familiar em salários mínimos & & & 0,12 \\
\hline Alta (25\%) & 1,00 & & \\
\hline Média (50\%) & 1,26 & $1,09-1,46$ & \\
\hline Baixa (25\%) & 1,19 & $0,99-1,44$ & \\
\hline Escolaridade (anos de estudos) & & & 0,43 \\
\hline Alta (25\%) & 1,00 & & \\
\hline Média (50\%) & 0,81 & $0,70-0,94$ & \\
\hline Baixa (25\%) & 0,88 & $0,73-1,07$ & \\
\hline Sexo & & & 0,004 \\
\hline Feminino & 1,00 & & \\
\hline Masculino & 1,21 & $1,08-1,35$ & \\
\hline Idade (anos) & & & 0,15 \\
\hline $20-29$ & 1,01 & & \\
\hline $30-39$ & 0,99 & $0,82-1,20$ & \\
\hline $40-49$ & 1,19 & $1,00-1,43$ & \\
\hline $50-59$ & 1,25 & $1,04-1,51$ & \\
\hline$\geq 60$ & 1,08 & $0,88-1,32$ & \\
\hline Estado civil & & & 0,89 \\
\hline Casado & 1,00 & & \\
\hline Solteiro & 1,05 & $0,92-1,20$ & \\
\hline Outros & 1,00 & $0,85-1,16$ & \\
\hline
\end{tabular}

IC95\%: intervalo de 95\% de confiança.

* Variáveis ajustadas entre si. 
Local de consulta conforme renda familiar com indivíduos de 20 a 69 anos, residentes na zona urbana do Município de São Leopoldo, Rio Grande do Sul, Brasil, 2006-2007.

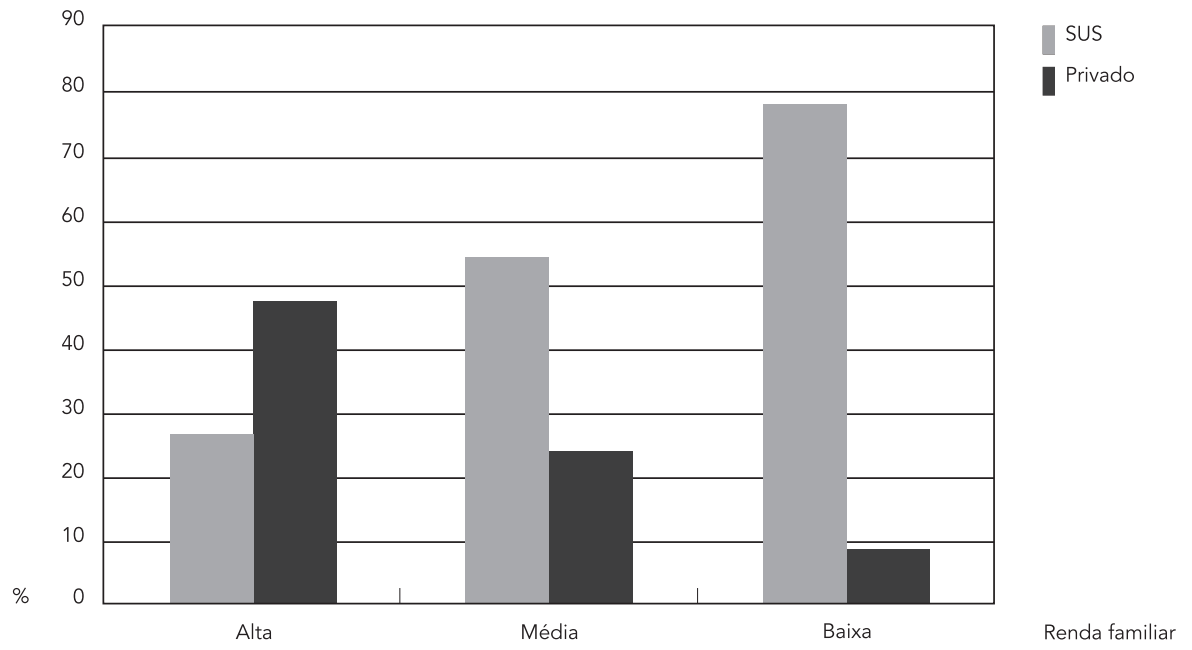

familiar classificada como média (valor de $\mathrm{p}<$ 0,001) e pertencentes à classe C 47,7\% (valor de $\mathrm{p}<0,001$ ). As variáveis cor da pele e idade não apresentaram associação com a realização de consultas pelo SUS. Após o ajuste das varáveis entre si, mantiveram associação estatística a renda familiar, com uma prevalência duas vezes maior entre aqueles com rendimentos menores do que os com renda alta. Os indivíduos com escolaridades média e baixa também apresentaram maiores prevalências de consultas no SUS, assim como os entrevistados classificados nas classes econômicas D e E (Tabela 3).

Em relação aos indivíduos que se consultaram com médico no último mês nos serviços privados de saúde, somente renda familiar, escolaridade e classe econômica pela ABEP apresentaram associação estatística. Apenas $8,1 \%$ dos entrevistados de baixa renda acessaram os serviços privados de saúde. Observou-se maior prevalência de consultas ao sistema privado entre os indivíduos de escolaridade elevada (64,1\%). A análise da classe econômica ABEP, mostrou que a prevalência de consultas nos serviços privados foram $43,7 \%$ nas classes A e B e apenas $11 \%$ nas classes D e E. As variáveis sexo, idade, cor da pele e estado civil não apresentaram associação estatística com a realização de consultas privadas. A análise ajustada mostrou que os indivíduos com renda familiar média e baixa se consultaram me- nos no setor privado. O grupo classificado como de baixa escolaridade apresentou uma prevalência de consultas no serviço privado de saúde $15 \%$ menor do que a classe alta, e os indivíduos inseridos nas classes econômicas D e E se consultaram $11 \%$ menos (Tabela 4).

\section{Discussão}

Este estudo ampliou as informações sobre o uso de serviços de saúde em São Leopoldo, na medida em que incluiu a população masculina. As informações até então disponíveis referiam-se a outro estudo transversal, realizado em 2003, que incluiu exclusivamente a população feminina 14 . Vale destacar que a coleta de dados de ambos os estudos foi realizada nos mesmos setores censitários e que a distribuição por idade, renda e escolaridade destes locais foi semelhante àquela verificada no censo para São Leopoldo. Entretanto, a pesquisa incluiu as pessoas reconhecidas como responsáveis pelo domicílio presentes no momento da entrevista, o que pode explicar o desvio observado na proporção de homens. Outros estudos transversais também constataram uma maior proporção de mulheres 11,12,13.

Pesquisas sobre a utilização de serviços de saúde têm empregado medidas diferentes quanto à temporalidade. No presente estudo, utilizou- 


\section{Tabela 3}

Prevalências de consultas médicas no SUS no último mês e análise ajustada usando-se a regressão de Poisson, com variáveis socioeconômicas e demográficas em indivíduos de 20 a 69 anos, residentes na zona urbana do Município de São Leopoldo, Rio Grande do Sul, Brasil, 2006-2007.

\begin{tabular}{|c|c|c|c|c|c|}
\hline \multirow[t]{2}{*}{ Variável } & \multicolumn{2}{|c|}{$\begin{array}{l}\text { Prevalência de consulta } \\
\text { no SUS }\end{array}$} & \multirow{2}{*}{$\begin{array}{c}\text { Razão de } \\
\text { prevalência } \\
\text { ajustada * }\end{array}$} & \multirow[t]{2}{*}{ IC95\% } & \multirow[t]{2}{*}{ Valor de $p$} \\
\hline & $\mathrm{n}$ & $\%$ & & & \\
\hline Renda familiar em salários mínimos & & & & & $<0,001$ \\
\hline Alta $(25 \%)$ & 32 & 24,8 & 1,00 & & \\
\hline Média (50\%) & 123 & 53,2 & 1,55 & $1,10-1,19$ & \\
\hline Baixa (25\%) & 24 & 78,4 & 2,12 & $1,47-3,06$ & \\
\hline Escolaridade (anos de estudos) & & & & & 0,009 \\
\hline Alta $(25 \%)$ & 7 & 10,9 & 1,00 & & \\
\hline Média (50\%) & 176 & 55,3 & 3,56 & $1,75-7,27$ & \\
\hline Baixa (25\%) & 56 & 63,6 & 3,26 & $1,57-6,79$ & \\
\hline Classe econômica & & & & & 0,014 \\
\hline$A-B$ & 44 & 29,1 & 1,00 & & \\
\hline C & 114 & 54,5 & 1,14 & $0,85-1,53$ & \\
\hline D-E & 81 & 74,3 & 1,40 & $1,03-1,90$ & \\
\hline Sexo & & & & & 0,5 \\
\hline Feminino & 194 & 53,2 & 1,00 & & \\
\hline Masculino & 55 & 45,1 & 1,05 & $0,85-1,30$ & \\
\hline
\end{tabular}

IC95\%: intervalo de 95\% de confiança.

* Variáveis ajustadas entre si.

Tabela 4

Prevalências de consultas médicas no setor privado no último mês e análise ajustada usando-se a regressão de Poisson, com variáveis socioeconômicas e demográficas. São Leopoldo, Rio Grande do Sul, Brasil, 2006-2007.

\begin{tabular}{|c|c|c|c|c|c|}
\hline \multirow[t]{2}{*}{ Variável } & \multicolumn{2}{|c|}{$\begin{array}{l}\text { Prevalência de consulta } \\
\text { no setor privado }\end{array}$} & \multirow{2}{*}{$\begin{array}{c}\text { Razão de } \\
\text { prevalência } \\
\text { ajustada * }\end{array}$} & \multirow[t]{2}{*}{ IC95\% } & \multirow[t]{2}{*}{ Valor de $p$} \\
\hline & $\mathrm{n}$ & $\%$ & & & \\
\hline Renda familiar & & & & & $<0,001$ \\
\hline Alta & 59 & 45,7 & 1,00 & & \\
\hline Média & 59 & 25,5 & 0,93 & $0,86-1,02$ & \\
\hline Baixa & 9 & 8,1 & 0,82 & $0,75-0,91$ & \\
\hline Escolaridade & & & & & 0,028 \\
\hline Alta & 41 & 64,1 & 1,00 & & \\
\hline Média & 71 & 22,3 & 0,81 & $0,73-0,89$ & \\
\hline Baixa & 18 & 20,5 & 0,85 & $0,76-0,96$ & \\
\hline \multicolumn{6}{|c|}{ Classe econômica } \\
\hline$A-B$ & 66 & 43,7 & 1,00 & & 0,036 \\
\hline$C$ & 51 & 24,4 & 0,97 & $0,89-1,05$ & \\
\hline D-E & 12 & 11,0 & 0,89 & $0,81-0,98$ & \\
\hline
\end{tabular}

IC95\%: intervalo de 95\% de confiança.

* Análise ajustada entre todas as variáveis. 
se "consulta médica no último mês", o que dificulta problemas de recordatório decorrentes de períodos mais prolongados 16 .

Com base na prevalência de consultas médicas observadas seria possível estimar que se o porcentual fosse mantido, a cada dois meses aproximadamente toda a população de São Leopoldo receberia, pelo menos, uma consulta médica, equivalendo a seis consultas/ano.

A análise das variáveis demográficas mostrou resultados condizentes com outras pesquisas semelhantes. As mulheres e as pessoas a partir de 60 anos se consultaram mais. Outros estudos nacionais tinham mostrado que as mulheres se consultaram mais do que os homens 7,8. Tem sido constatado que as pessoas idosas necessitam de mais cuidados de saúde por apresentarem múltiplas morbidades, conforme relatados em estudos brasileiros 9,17,18,19,20,21.

Entretanto, no presente estudo um achado foi relevante. Na análise entre "quem não consultou”, não foram encontradas diferenças entre as variáveis socioeconômicas nas categorias extremas. A análise evidenciou que os indivíduos nas categorias intermediárias de renda, escolaridade e classe econômica se consultavam menos. Estudos realizados no Brasil têm mostrado diferenças entre o uso de serviços de saúde e condições socioeconômicas, sempre constatando que os grupos sociais menos favorecidos têm menor utilização de serviços de saúde. Deve-se destacar que os indivíduos das classes D e E, e até mesmo as pessoas inseridas nas categorias intermediárias se consultam menos 7,9,20. Esses achados são par- ticularmente importantes em relação à saúde das mulheres, no que diz respeito a exames de detecção precoce de afecções como câncer de colo uterino e de mama. Outros estudos, no entanto, revelaram que a implantação da Estratégia Saúde da Família (ESF) pode atenuar essas desigualdades 21,22. Contudo, em São Leopoldo a cobertura da ESF não pode ser considerada elevada, atingindo $18 \%$. Porém, o achado pode ser explicado pelo elevado número de consultas médicas. Uma possível explicação para o fato, portanto, seria o de que as classes extremas estariam cobertas pelo setor privado, no caso das classes A e B e de renda familiar alta, e pelo SUS no caso das classes $\mathrm{D}$ e E e de renda familiar baixa. A classe intermediária não possuindo "capacidade de compra" para ingresso nos serviços privados 23 e acesso ao público ${ }^{24}$ apresentaria uma maior predisposição a não consulta.

O presente estudo revelou também que o local de consulta independe das condições demográficas, sendo claramente determinado pelas variáveis socioeconômicas. Outros estudos realizados em Pelotas também tinham constatado essas diferenças, mostrando que os indivíduos de melhor nível socioeconômico preferencialmente recorreram aos serviços privados, enquanto o padrão de utilização das pessoas mais pobres é o SUS 25,26.

Por fim, ressalta-se a importância da realização de estudos acerca da utilização de serviços de saúde, tendo em vista a sua capacidade para detectar iniquidades no sistema de saúde e contribuir para o seu contínuo aperfeiçoamento.

\footnotetext{
Resumo

O objetivo do estudo foi descrever as características da população adulta em São Leopoldo, Rio Grande do Sul, Brasil, quanto ao uso de serviços de saúde. Avaliaramse como desfecho: não se consultar com médico no último mês, utilizar serviços do SUS e se consultar nos serviços privados. Foi realizado um estudo transversal com indivíduos de ambos os sexos, de 20 a 69 anos. Das 1.098 pessoas respondentes, 623 (56,7\%; IC95\%: 53,8-59,7) não haviam se consultado com médico no último mês. Dos 487 indivíduos que tinham se consultado, 51,2\% utilizaram os serviços do SUS, 26,9\% os serviços privados e 22\% outros serviços. Consultar
}

estava associado com sexo feminino e idade elevada. A análise evidenciou que os indivíduos nas categorias intermediárias de renda, escolaridade e classe econômica se consultavam menos do que as correspondentes altas e baixas categorias. Os resultados sugerem que a classe intermediária, por não possuir "capacidade de compra" para serviços privados elou por não utilizar o sistema público, tenderia a procurar assistência de saúde com menor frequência.

Acesso aos Serviços de Saúde; Avaliação em Saúde; Equidade em Saúde 


\section{Colaboradores}

J. S. Dias-da-Costa, S. A. Soares, M. F. Nunes, T. Bagatini, M. C. Marques, L. K. Guimarães, L. P. Müller, F. C. S. Machado e E. S. Barcellos, participaram da revisão bibliográfica, análise de dados e redação do artigo. M. T. A. Olinto colaborou na análise dos dados e revisão do texto final. M. P. Pattussi contribuiu na elaboração do projeto, análise dos dados e redação do artigo.

\section{Agradecimentos}

Projeto financiado pelo Conselho Nacional de Desenvolvimento Científico e Tecnológico (CNPq, processo $n^{\circ}$. 478503/2004-0), e pela Fundação de Amparo à Pesquisa do Estado do Rio Grande do Sul (FAPERGS, processo $\mathrm{n}^{\circ}$. 0415621).

\section{Referências}

1. Andersen RM. Revisiting the behavioral model and access to medical care: does it matter? J Health Soc Behav 1995; 36:1-10.

2. Campbell SM, Roland MO, Buetow SA. Defining quality of care. Soc Sci Med 2000; 51:1611-25.

3. Marmot M. Health in an unequal world. Lancet 2006; 368:2081-94.

4. Starfield B, Shi L, Macinko J. Contribution of primary care to health systems and health. Milbank Q 2005; 83:457-502.

5. Travassos C, Martins M. Uma revisão sobre os conceitos de acesso e utilização de serviços de saúde. Cad Saúde Pública 2004; 20 Suppl 2:S190-8.

6. Santos IS, Victora CG. Serviços de saúde: epidemiologia, pesquisa e avaliação. Cad Saúde Pública 2004; 20 Suppl 2:S337-41.

7. Travassos C, Viacava F, Pinheiro R, Brito A. Utilização dos serviços de saúde no Brasil: gênero, características familiares e condição social. Rev Panam Salud Pública 2002; 11:365-73.
8. Pinheiro RS, Viacava F, Travassos V, Brito AS. Gênero, morbidade, acesso e utilização de serviços de saúde no Brasil. Ciênc Saúde Coletiva 2002; 7 : 687-707.

9. Noronha KVMS, Andrade MV. Desigualdades sociais em saúde e na utilização dos serviços de saúde entre os idosos na América Latina. Rev Panam Salud Pública 2005; 17:410-8.

10. Travassos C, Oliveira EXG, Viacava F. Desigualdades geográficas e sociais no acesso aos serviços de saúde no Brasil: 1998 e 2003. Ciênc Saúde Coletiva 2006; 11:975-86.

11. Mendoza-Sassi R, Béria JU, Barros AJD. Outpatient health service utilization and associated factors: a population-based study. Rev Saúde Pública 2003; 37:372-8.

12. Capilheira MF, Santos IS. Individual factors associated with medical consultation by adults. Rev Saúde Pública 2006; 40:436-43. 
13. Dias-da-Costa JS, Reis MC, Silveira Filho CV, Linhares RS, Piccinini F. Prevalência de consultas médicas e fatores associados, Pelotas - RS, 19992000. Rev Saúde Pública 2008; 42:1074-84.

14. Dias-da-Costa JS, Presser AD, Zanotta AF, Ferreira DG, Perozzo G, Freitas IBA, et al. Utilização dos serviços ambulatoriais de saúde por mulheres: estudo de base populacional no Sul do Brasil. Cad Saúde Pública 2008; 24:2843-51.

15. Barros AJD, Hirakata UM. Alternatives for logistic regression in cross-sectional studies: an empirical comparision of models that directly estimate the prevalence ratio. BMC Med Res Methodol 2003; 3:21.

16. Nordberg E. Household health surveys in developing countries: could more use be made of them in planning? Health Policy Plan 1988; 3:32-9.

17. Gomes R, Nascimento EF, Araujo FC. Por que os homens buscam menos os serviços de saúde do que as mulheres? As explicações de homens com baixa escolaridade e homens com ensino superior. Cad Saúde Pública 2007; 23:565-74.

18. Barros MBA, Chester LG, Carandina L, Dalla Torre G. Desigualdades sociais na prevalência de doenças crônicas no Brasil. PNAD 2003. Ciênc Saúde Coletiva 2006; 11:911-26.

19. Lima-Costa MF, Barreto SM, Giatti L. Condições de saúde, capacidade funcional, uso de serviços de saúde e gastos com medicamentos da população idosa brasileira: um estudo descritivo baseado na Pesquisa Nacional por Amostras de Domicílios. Cad Saúde Pública 2003; 19:735-43.
20. Barata RB, Almeida MF, Montero CV, Silva ZP. Health inequalities on ethnicity in individuals aged 15 to 64, Brazil, 1998. Cad Saúde Pública 2007; 23:305-13.

21. Fernandes LCL, Bertoldi AD, Barros AJD. Utilização dos serviços de saúde pela população coberta pela Estratégia de Saúde da Família. Rev Saúde Pública 2009; 43:595-603.

22. Goldbaum M, Gianini RJ, Novaes HMD, César CLG. Utilização de serviços de saúde em áreas cobertas pelo programa de saúde da família (Qualis) no Município de São Paulo. Rev Saúde Pública 2005; 39:90-9.

23. Donabedian A. Aspects of medical care administration. Boston: Harvard University Press; 1973.

24. Penchansky DBA, Thomas JW. The concept of access: definition and relationship to consumer satisfaction. Med Care 1981; 19:127-40.

25. Dias-da-Costa JS, Facchini LA. Utilização de serviços ambulatoriais em Pelotas: onde a população consulta? Com que freqüência? Rev Saúde Pública 1997; 31:360-9.

26. Dias-da-Costa JS, Gigante DP, Horta BL, Barros FC, Victora CG. Utilização de serviços de saúde por adultos da coorte de nascimentos de 1982 a 2004-5, Pelotas, RS. Rev Saúde Pública 2008; 42 Suppl 2:51-9.

Recebido em 12/Jan/2010

Versão final reapresentada em 01/Mar/2011

Aprovado em 11/Mar/2011 